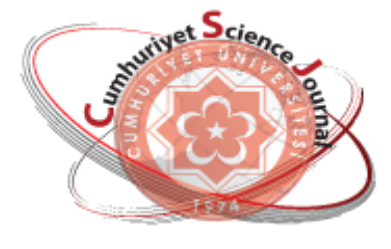

e-ISSN: $2587-246 X$

ISSN: $2587-2680$

\section{Cundenory at Seionce Journal esy}

\author{
Cumhuriyet Sci. J., Vol.40-2(2019) 396-405
}

\title{
Investigation of Benzimidazole Derivates as Corrosion Inhibitor by DFT
}

\author{
Burak TüZZ̈U $N^{1, *}$ \\ ${ }^{1}$ Science Faculty, Department of Chemistry, Cumhuriyet University, Sivas 58140, Turkey
}

Received: 04.04.2018; Accepted: 17.12.2018

http://dx.doi.org/10.17776/csj.412611

\begin{abstract}
Benzimidazole derivates are investigated the activity of corrosion inhibitor molecules.In quantum chemical calculation, different parameters such as $\mathrm{E}_{\text {HOмо, }} \mathrm{E}_{\mathrm{LumO}} \Delta \mathrm{E}$ (HOMO-LUMO energy gap), electronegativity, chemical hardness, global softness, nucleophilicity are calculated by Gaussian 09 software. Studied molecules were performed using the Hartree-Fock (HF) and Becke, 3-parameter, Lee-Yang-Parr (B3LYP) method with sdd, cep-4g, 3-21G, 6-31G, 6-31++G, lanl2dz basis set in gas and aqueous phase. We can see the corrosion inhibitor ranking as: $4 \mathrm{NPBI}>4 \mathrm{APBI}>2 \mathrm{NPBI}>2 \mathrm{APBI}>4 \mathrm{BPBI}>4 \mathrm{MPBI}>4 \mathrm{CPBI}>$ PBI in B3lyp method with sdd and lanl2dz.
\end{abstract}

Keywords: Benzimidazole, DFT, corrosion, activity.

\section{DFT ile benzimidazol türevlerinin korozyon inhibitörü olarak incelenmesi}

Özet. Benzimidazol türevleri, korozyon önleyici moleküllerin aktivitesini araştırıldı. Kuantum kimyasal hesaplamasında, $\mathrm{E}_{\text {номо, }} \mathrm{E}_{\mathrm{LUMO}}, \Delta \mathrm{E}$ (HOMO-LUMO enerji aralığı), elektronegatiflik, kimyasal sertlik, global yumuşaklık, nükleofilik gibi farklı parametreler gaussian 09 yazılımı ile hesaplandı. Çalışılan moleküller, Hartree-Fock (HF) ve Becke, 3-parametre Lee-Yang-Parr (B3LYP) yöntemi kullanılarak sdd, cep-4g, 3-21G, 6-31G, 6-31 ++ G, lanl2dz temel sette gaz ve sulu fazda hesaplamaları yapıldı. Korozyon inhibitörü sıralamasını aşağıdaki gibi görebiliriz: 4NPBI $>4 \mathrm{APBI}>2 \mathrm{NPBI}>2 \mathrm{APBI}>4 \mathrm{BPBI}>4 \mathrm{MPBI}>4 \mathrm{CPBI}>\mathrm{PBI}$, b3lyp metodunda lanl $2 \mathrm{dz}$ ve sdd settinde.

Anahtar Kelimeler: Benzimidazol, DFT, korozyon, aktivite.

\section{INTRODUCTION}

Metal corrosion is a very important problem in various industrial processes which is widely used water, alcohol and acid. The acid solutions used cause too much corrosion in the metal that is an iron, copper, aluminum. Corrosion inhibitors that are containing nitrogen, oxygen, Sulphur and aromatic ring, are used to prevent corrosion caused by acid solutions.

As it is well known that experimental studies have been used to understand the corrosion inhibition mechanism of molecules and to explain corrosion inhibition efficiencies. Quantum chemical calculations provide preliminary information on the activities of molecules. In quantum chemical calculations, parameters related to the activity of molecules are calculated using density functional theory (DFT) that are calculated HOMO (highest occupied molecular orbital), LUMO (lowest unoccupied molecular orbital), electrophilicity, electronegativity, chemical potential, chemical hardness and nucleophilicity. 
In this study, we can be seen that activity of studied molecules whose names are 2-(4-nitrophenyl) benzimidazole (4NPBI), 2-(4-aminophenyl) benzimidazole (4APBI), 2-(2-nitrophenyl) benzimidazole (2NPBI), 2-(2-aminophenyl) benzimidazole (2APBI), 2-phenyl benzimidazole (PBI), 2-(4-chlorophenyl) benzimidazole (4CPBI), 2-(4-metilphenyl) benzimidazole (4MPBI), 2-(4bromophenyl) benzimidazole (4BPBI) in Figure 1 [1].

\section{COMPUTATIONAL DETAILS}

DFT calculation is the most popular method for the activity of molecules.In this study, we prepared the input files of the molecules studied by gaussian view 5.08 programs [2]. Calculations of studied molecules were performed with Gaussian IA32WG09RevA.02 and Gaussian AS64L-G09RevD.01 programs [3-4]. Studied molecules were performed using the Hartree-Fock (HF)[5] and Becke, 3parameter, Lee-Yang-Parr (B3LYP) [6-8] method with sdd, cep-4g, 3-21G, 6-31G, 6-31++G, lanl2dz basis set in gas and an aqueous phase. HOMO and LUMO are given information about activity of molecules.Chemical reactivity parameter of molecules isgiven to found a good corrosion inhibitor such as $\mathrm{E}_{\text {номо, }} \mathrm{E}_{\mathrm{LUMO}}, \Delta \mathrm{E}$ (HOMOLUMO energy gap), electronegativity ( $\chi)$, chemical potential $(\mu)$, chemical hardness $(\eta)$, electrophilicity $(\omega)$, nucleophilicity $(\varepsilon)$, global softness $(\sigma)$ and proton affinity (PA) [9-18].

$$
\begin{aligned}
& \mu=-\chi=\left(\frac{\partial E}{\partial N}\right)_{v(r)} \\
& \eta=\frac{1}{2}\left(\frac{\partial^{2} E}{\partial N^{2}}\right)_{v(r)}=\frac{1}{2}\left(\frac{\partial \mu}{\partial N}\right)
\end{aligned}
$$

Ionization energy (I) and electron affinity (A) [19] of studied molecules are calculated with HOMO and LUMO energy that are interested Electronegativity, global softness and chemical hardness obtaining the following equations.

$$
\chi=-\mu=\left(\frac{I+A}{2}\right)
$$

$$
\eta=\frac{I-A}{2}
$$

As it is well known that global softness is defined as the inverse of the chemical hardness [20].

$\sigma=1 / \eta$

$\chi=-\mu=\left(\frac{-E_{H O M O}-E_{L U M O}}{2}\right)$

$\eta=\left(\frac{E_{L U M O}-E_{\text {HOMO }}}{2}\right)$

The global electrophilicity index $(\omega)$ that is investigated by Parr et al., is the inverse of nucleophilicity and are given inequality (8). Electrophilicity and nucleophilicity are used for the prediction organic and inorganic reaction mechanisms. Nucleophilicity $(\varepsilon)$ is defined as the inverse of the electrophilicity in equations (9).

$$
\begin{aligned}
& \omega=\mu^{2} / 2 \eta=\chi^{2} / 2 \eta \\
& \varepsilon=1 / \omega
\end{aligned}
$$

\section{RESULTS AND DISCUSSION}

The corrosion inhibitor reactivity of benzimidazole derivatives is studied by quantum chemical calculation. The chemical reactivity of studied molecules that is obtained by the Gaussian software program, it is shown that a good inhibitor against different metal atoms. The studied of molecules are obtained the results are given in below.

The studied molecules are investigated quantum chemical parameter such as $\mathrm{E}_{\text {Hомо, }} \mathrm{E}_{\mathrm{LumO}}, \Delta \mathrm{E}$ (HOMO-LUMO energy gap), electronegativity, chemical potential, chemical hardness, electrophilicity, nucleophilicity, global softness and proton affinity. These parameters are very important parameters which are compared the reactivity of studied molecules.

As it is well known that the properties of chemical reactivity of studied molecules were compared by molecular orbitals of an inhibitor molecule that have got two molecular orbitalswhose the name is 
Highest Occupied Molecular Orbital (HOMO) and Lowest Unoccupied Molecular Orbital (LUMO).

The energy level of HOMO has defined the electron donating ability of studied molecules. The molecule has got the high values of energy of HOMO that is showingthe tendency to donate electrons of the molecule to appropriate that the acceptor molecules have low energy and empty molecular orbital [9-18]. From the light of the<smiles>O=[N+]([O-])c1ccc(-c2nc3ccccc3[nH]2)cc1</smiles><smiles>O=[N+]([O-])c1ccccc1-c1nc2ccccc2[nH]1</smiles><smiles>c1ccc(-c2nc3ccccc3[nH]2)cc1</smiles>

PBI

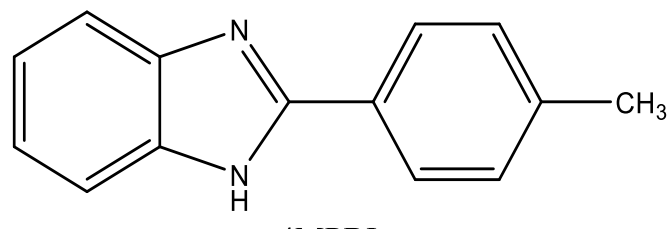

4MPBI result given in the information, the energy level of LUMO of molecules is indicated electron accepting abilities of studied molecules. When the energy value of LUMO of inhibitor molecule is lower, this molecule has more electron accepting ability in lower energy of molecular orbitals. The calculated of HOMO and LUMO energy value is given in Table 1, 2, 3 and 4.<smiles>Nc1ccc(-c2nc3ccccc3[nH]2)cc1</smiles><smiles>Nc1ccccc1-c1nc2ccccc2[nH]1</smiles>

2APBI

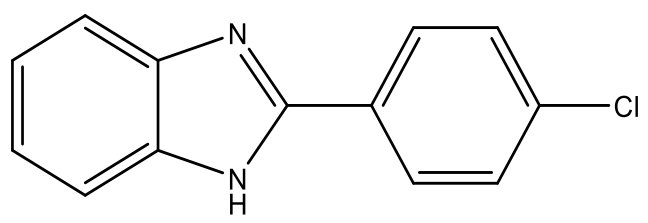

4CPBI

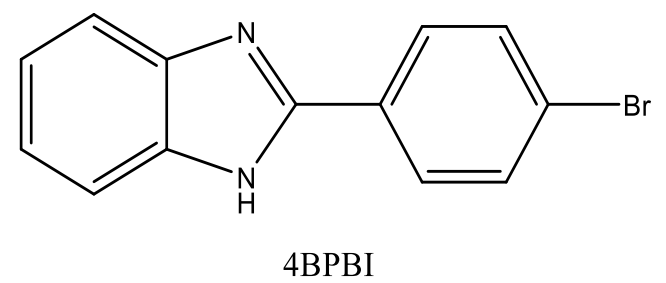

Figure 1. The structure and schematic representation of molecules of benzimidazole derivatives

In figure 2, structure of HOMO, LUMO and ESP studied molecules are given about some information of this molecule. In figure of HOMO of molecule, we look where highest occupied molecular orbital is. In this picture, HOMO orbitals appear to cover the entire molecule. In figure of LUMO of molecule, we look where lowest unoccupied molecular orbital is. In this picture, LUMO orbitals appear to cover the entire molecule. Last picture is ESP that is Molecular electrostatic potential (ESP) figure that given information about distribution of electrons in molecular. In this picture, the different value of the electrostatic potential represented by different colors [14]. This potential increases in the order red $>$ orange $>$ yellow $>$ green $>$ blue. The highest potential is on oxygen atoms.

The energy gap value $(\Delta \mathrm{E})$ in chemical reactivity of inhibitor molecule is a very important parameter in corrosion. As it is well known that inhibitor molecule has a small energy gap value, this molecule is a good corrosion inhibitor. Since the energy gap value of inhibitor molecule is indicated 
that the binding ability of inhibitor molecules on metal surfaces [21]. On the basis of the calculated the energy gap value given in Table 1, 2, 3 and 4, the corrosion inhibition activity of benzimidazole derivatives molecules can be written as:4NPBI >
$4 \mathrm{APBI}>2 \mathrm{NPBI}>2 \mathrm{APBI}>4 \mathrm{BPBI}>4 \mathrm{MPBI}>$ 4CPBI > $\mathrm{PBI}$ in B3lyp method with sdd and lanl2dz.

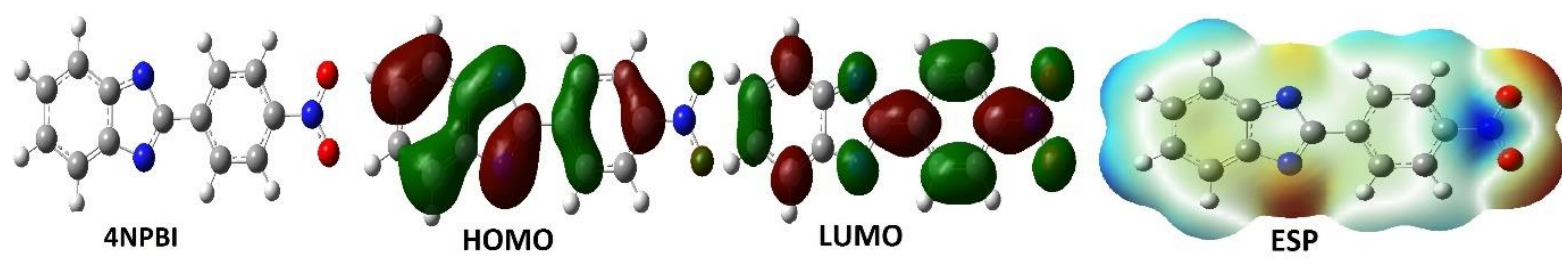

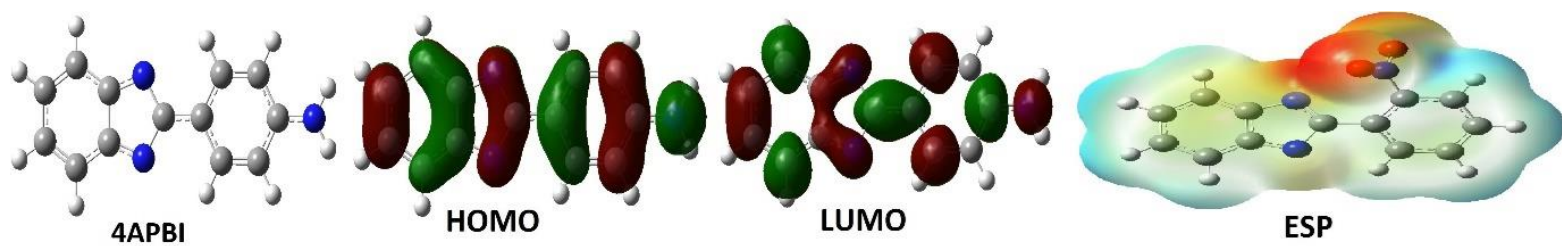
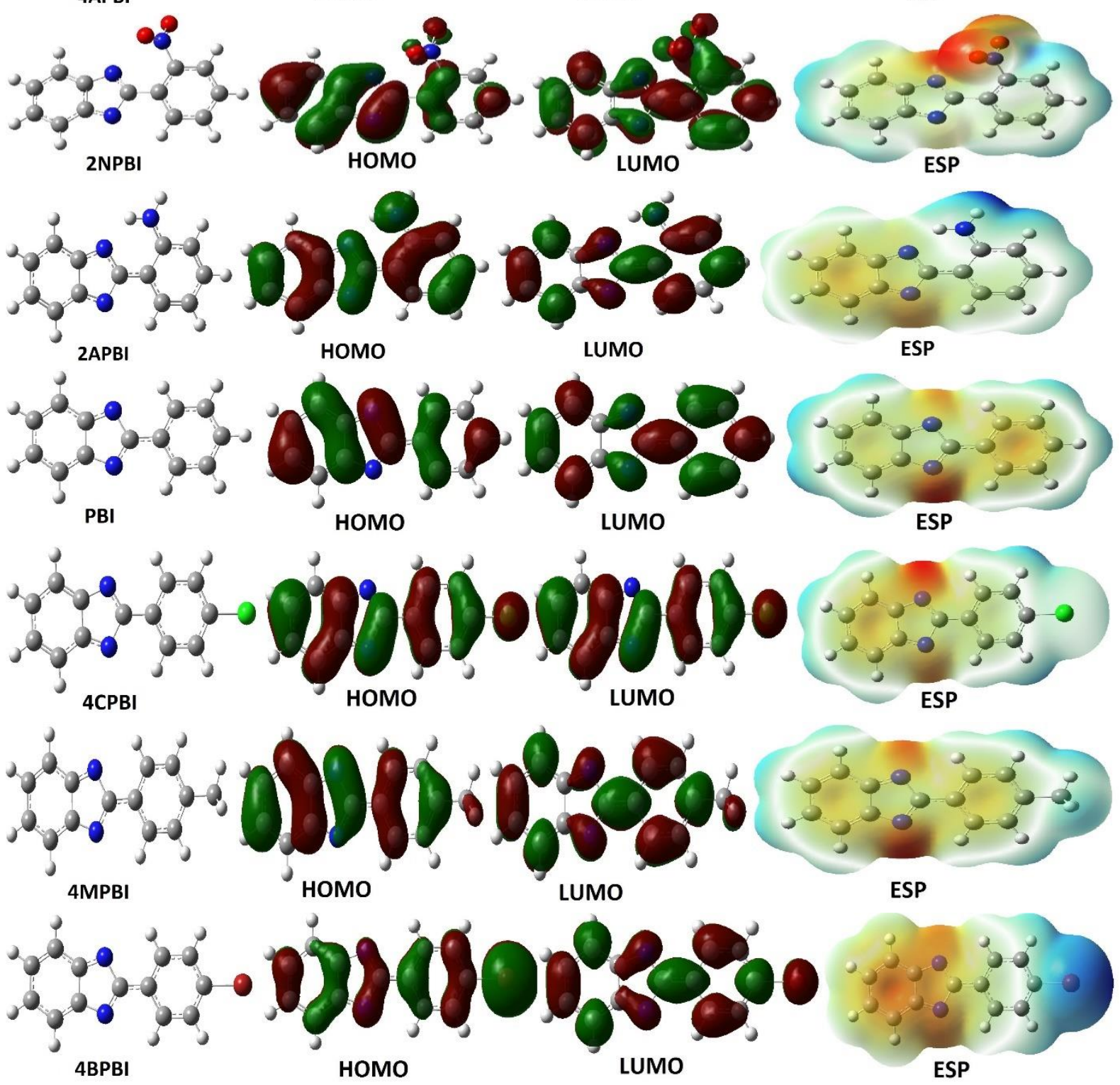

Figure 2. Structures of HOMO, LUMO and ESPs of benzimidazole derivatives 
Table 1. The calculated quantum chemical parameters with B3LYP method in gas phase (eV)

\begin{tabular}{|c|c|c|c|c|c|c|c|c|c|c|c|c|c|}
\hline & Еномо & ELUMo & I & $\mathbf{A}$ & $\Delta \mathbf{E}$ & $\eta$ & $\sigma$ & $\chi$ & $\mathbf{P I}$ & $\omega$ & $\varepsilon$ & dipol & Energy \\
\hline \multicolumn{14}{|c|}{ B3LYP/SDD } \\
\hline 4NPBI & $-6,903$ & $-3,324$ & 6,903 & 3,324 & 3,579 & 1,790 & 0,559 & 5,113 & $-5,113$ & 7,305 & 0,137 & 8,811 & $-22163,416$ \\
\hline 4APBI & $-5,376$ & $-1,259$ & 5,376 & 1,259 & 4,117 & 2,059 & 0,486 & 3,317 & $-3,317$ & 2,673 & 0,374 & 5,861 & $-18105,389$ \\
\hline 2NPBI & $-6,701$ & $-2,566$ & 6,701 & 2,566 & 4,136 & 2,068 & 0,484 & 4,633 & $-4,633$ & 5,191 & 0,193 & 4,634 & $-22162,904$ \\
\hline 2APBI & $-5,618$ & $-1,471$ & 5,618 & 1,471 & 4,147 & 2,073 & 0,482 & 3,544 & $-3,544$ & 3,030 & 0,330 & 2,949 & $-18105,611$ \\
\hline PBI & $-6,193$ & $-1,703$ & 6,193 & 1,703 & 4,490 & 2,245 & 0,445 & 3,948 & $-3,948$ & 3,471 & 0,288 & 0,998 & $-16599,339$ \\
\hline 4CPBI & $-6,345$ & $-1,920$ & 6,345 & 1,920 & 4,425 & 2,213 & 0,452 & 4,132 & $-4,132$ & 3,858 & 0,259 & 3,522 & $-29105,350$ \\
\hline 4MPBI & $-6,009$ & $-1,597$ & 6,009 & 1,597 & 4,413 & 2,206 & 0,453 & 3,803 & $-3,803$ & 3,277 & 0,305 & 0,391 & $-17668,447$ \\
\hline 4BPBI & $-6,337$ & $-1,939$ & 6,337 & 1,939 & 4,398 & 2,199 & 0,455 & 4,138 & $-4,138$ & 3,893 & 0,257 & 3,421 & $-16947,196$ \\
\hline \multicolumn{14}{|c|}{ B3LYP/Cep-4g } \\
\hline 4NPBI & $-8,192$ & $-4,904$ & 8,192 & 4,904 & 3,288 & 1,644 & 0,608 & 6,548 & $-6,548$ & 13,041 & 0,077 & 8,251 & $-3762,591$ \\
\hline 4APBI & $-6,682$ & $-3,025$ & 6,682 & 3,025 & 3,657 & 1,829 & 0,547 & 4,853 & $-4,853$ & 6,440 & 0,155 & 3,823 & $-2932,975$ \\
\hline 2NPBI & $-8,018$ & $-4,466$ & 8,018 & 4,466 & 3,552 & 1,776 & 0,563 & 6,242 & $-6,242$ & 10,968 & 0,091 & 4,138 & $-3762,160$ \\
\hline 2APBI & $-6,936$ & $-3,193$ & 6,936 & 3,193 & 3,743 & 1,872 & 0,534 & 5,065 & $-5,065$ & 6,852 & 0,146 & 2,577 & $-2933,299$ \\
\hline PBI & $-7,652$ & $-3,443$ & 7,652 & 3,443 & 4,209 & 2,105 & 0,475 & 5,547 & $-5,547$ & 7,310 & 0,137 & 1,906 & $-2649,286$ \\
\hline 4CPBI & $-7,793$ & $-3,739$ & 7,793 & 3,739 & 4,054 & 2,027 & 0,493 & 5,766 & $-5,766$ & 8,201 & 0,122 & 4,731 & $-3038,153$ \\
\hline 4MPBI & $-7,498$ & $-3,327$ & 7,498 & 3,327 & 4,171 & 2,085 & 0,480 & 5,413 & $-5,413$ & 7,024 & 0,142 & 0,913 & $-2833,735$ \\
\hline 4BPBI & $-7,507$ & $-3,506$ & 7,507 & 3,506 & 4,001 & 2,000 & 0,500 & 5,507 & $-5,507$ & 7,579 & 0,132 & 2,813 & $-2997,697$ \\
\hline \multicolumn{14}{|c|}{ B3LYP/3-21g } \\
\hline 4NPBI & $-6,653$ & $-2,814$ & 6,653 & 2,814 & 3,839 & 1,920 & 0,521 & 4,733 & $-4,733$ & 5,836 & 0,171 & 7,293 & $-22043,816$ \\
\hline 4APBI & $-5,108$ & $-0,876$ & 5,108 & 0,876 & 4,231 & 2,116 & 0,473 & 2,992 & $-2,992$ & 2,116 & 0,473 & 6,020 & $-18008,316$ \\
\hline 2NPBI & $-6,514$ & $-2,448$ & 6,514 & 2,448 & 4,067 & 2,033 & 0,492 & 4,481 & $-4,481$ & 4,938 & 0,203 & 4,463 & $-22043,329$ \\
\hline 2APBI & $-5,362$ & $-1,146$ & 5,362 & 1,146 & 4,216 & 2,108 & 0,474 & 3,254 & $-3,254$ & 2,511 & 0,398 & 3,212 & $-18008,695$ \\
\hline PBI & $-6,055$ & $-1,394$ & 6,055 & 1,394 & 4,660 & 2,330 & 0,429 & 3,724 & $-3,724$ & 2,977 & 0,336 & 1,122 & $-16510,517$ \\
\hline 4CPBI & $-6,256$ & $-1,660$ & 6,256 & 1,660 & 4,595 & 2,298 & 0,435 & 3,958 & $-3,958$ & 3,409 & 0,293 & 3,943 & $-28957,276$ \\
\hline 4MPBI & $-5,886$ & $-1,311$ & 5,886 & 1,311 & 4,575 & 2,288 & 0,437 & 3,598 & $-3,598$ & 2,830 & 0,353 & 0,200 & $-17573,972$ \\
\hline 4BPBI & $-6,092$ & $-1,568$ & 6,092 & 1,568 & 4,524 & 2,262 & 0,442 & 3,830 & $-3,830$ & 3,242 & 0,308 & 2,607 & $-86205,409$ \\
\hline \multicolumn{14}{|c|}{ B3LYP/6-31g } \\
\hline 4NPBI & $-6,699$ & $-3,019$ & 6,699 & 3,019 & 3,681 & 1,840 & 0,543 & 4,859 & $-4,859$ & 6,414 & 0,156 & 8,360 & $-22160,493$ \\
\hline 4APBI & $-5,171$ & $-0,971$ & 5,171 & 0,971 & 4,200 & 2,100 & 0,476 & 3,071 & $-3,071$ & 2,246 & 0,445 & 5,850 & $-18103,585$ \\
\hline 2NPBI & $-6,499$ & $-2,190$ & 6,499 & 2,190 & 4,309 & 2,154 & 0,464 & 4,345 & $-4,345$ & 4,381 & 0,228 & 4,369 & $-22159,960$ \\
\hline 2APBI & $-5,420$ & $-1,196$ & 5,420 & 1,196 & 4,224 & 2,112 & 0,474 & 3,308 & $-3,308$ & 2,591 & 0,386 & 2,862 & $-18103,805$ \\
\hline PBI & $-6,008$ & $-1,428$ & 6,008 & 1,428 & 4,580 & 2,290 & 0,437 & 3,718 & $-3,718$ & 3,018 & 0,331 & 0,968 & $-16597,877$ \\
\hline 4CPBI & $-6,200$ & $-1,682$ & 6,200 & 1,682 & 4,519 & 2,259 & 0,443 & 3,941 & $-3,941$ & 3,437 & 0,291 & 3,765 & $-29103,991$ \\
\hline 4MPBI & $-5,838$ & $-1,343$ & 5,838 & 1,343 & 4,495 & 2,247 & 0,445 & 3,590 & $-3,590$ & 2,868 & 0,349 & 0,307 & $-17666,882$ \\
\hline 4BPBI & $-6,124$ & $-1,644$ & 6,124 & 1,644 & 4,479 & 2,240 & 0,446 & 3,884 & $-3,884$ & 3,368 & 0,297 & 3,096 & $-86558,506$ \\
\hline \multicolumn{14}{|c|}{ B3LYP/6-31++g } \\
\hline 4NPBI & $-6,977$ & $-3,443$ & 6,977 & 3,443 & 3,534 & 1,767 & 0,566 & 5,210 & $-5,210$ & 7,679 & 0,130 & 8,847 & $-22161,453$ \\
\hline 4APBI & $-5,483$ & $-1,352$ & 5,483 & 1,352 & 4,130 & 2,065 & 0,484 & 3,417 & $-3,417$ & 2,827 & 0,354 & 5,786 & $-18104,341$ \\
\hline 2NPBI & $-6,769$ & $-2,745$ & 6,769 & 2,745 & 4,025 & 2,012 & 0,497 & 4,757 & $-4,757$ & 5,623 & 0,178 & 4,581 & $-22160,962$ \\
\hline 2APBI & $-5,716$ & $-1,536$ & 5,716 & 1,536 & 4,180 & 2,090 & 0,478 & 3,626 & $-3,626$ & 3,145 & 0,318 & 2,846 & $-18104,506$ \\
\hline PBI & $-6,277$ & $-1,757$ & 6,277 & 1,757 & 4,520 & 2,260 & 0,442 & 4,017 & $-4,017$ & 3,571 & 0,280 & 0,942 & $-16598,523$ \\
\hline 4CPBI & $-6,440$ & $-1,981$ & 6,440 & 1,981 & 4,460 & 2,230 & 0,448 & 4,211 & $-4,211$ & 3,975 & 0,252 & 3,607 & $-29104,649$ \\
\hline 4MPBI & $-6,095$ & $-1,664$ & 6,095 & 1,664 & 4,431 & 2,216 & 0,451 & 3,879 & $-3,879$ & 3,396 & 0,294 & 0,456 & $-17667,551$ \\
\hline 4BPBI & $-6,385$ & $-1,962$ & 6,385 & 1,962 & 4,423 & 2,212 & 0,452 & 4,174 & $-4,174$ & 3,939 & 0,254 & 3,097 & $-86559,996$ \\
\hline \multicolumn{14}{|c|}{ B3LYP/Lanl2dz } \\
\hline 4NPBI & $-6,908$ & $-3,330$ & 6,908 & 3,330 & 3,578 & 1,789 & 0,559 & 5,119 & $-5,119$ & 7,323 & 0,137 & 8,827 & $-22163,371$ \\
\hline 4APBI & $-5,379$ & $-1,261$ & 5,379 & 1,261 & 4,117 & 2,059 & 0,486 & 3,320 & $-3,320$ & 2,677 & 0,374 & 5,862 & $-18105,360$ \\
\hline 2NPBI & $-6,706$ & $-2,574$ & 6,706 & 2,574 & 4,131 & 2,066 & 0,484 & 4,640 & $-4,640$ & 5,212 & 0,192 & 4,640 & $-22162,858$ \\
\hline 2APBI & $-5,620$ & $-1,473$ & 5,620 & 1,473 & 4,147 & 2,074 & 0,482 & 3,547 & $-3,547$ & 3,033 & 0,330 & 2,955 & $-18105,584$ \\
\hline PBI & $-6,196$ & $-1,705$ & 6,196 & 1,705 & 4,491 & 2,246 & 0,445 & 3,950 & $-3,950$ & 3,475 & 0,288 & 1,003 & $-16599,313$ \\
\hline 4CPBI & $-6,362$ & $-1,931$ & 6,362 & 1,931 & 4,430 & 2,215 & 0,451 & 4,147 & $-4,147$ & 3,881 & 0,258 & 3,656 & $-16989,793$ \\
\hline 4MPBI & $-6,013$ & $-1,598$ & 6,013 & 1,598 & 4,414 & 2,207 & 0,453 & 3,806 & $-3,806$ & 3,281 & 0,305 & 0,387 & $-17668,421$ \\
\hline 4BPBI & $-6,311$ & $-1,920$ & 6,311 & 1,920 & 4,391 & 2,195 & 0,455 & 4,116 & $-4,116$ & 3,858 & 0,259 & 3,211 & $-16941,355$ \\
\hline
\end{tabular}


Table 2. The calculated quantum chemical parameters with B3LYP method in aqueous phase (eV)

\begin{tabular}{|c|c|c|c|c|c|c|c|c|c|c|c|c|c|}
\hline \multirow{2}{*}{\multicolumn{14}{|c|}{$\frac{\text { Еномо }^{2}}{\text { B3LYP/SDD }}$}} \\
\hline & & & & & & & & & & & & & \\
\hline 4APBI & $-5,643$ & $-1,564$ & 5,643 & 1,564 & 4,079 & 2,040 & 0,490 & 3,604 & $-3,604$ & 3,184 & 0,314 & 11,666 & $-18105,943$ \\
\hline 2APBI & $-5,853$ & $-1,665$ & 5,853 & 1,665 & 4,188 & 2,094 & 0,478 & 3,759 & $-3,759$ & 3,375 & 0,296 & 6,213 & $-18105,980$ \\
\hline PBI & $-6,402$ & $-1,910$ & 6,402 & 1,910 & 4,492 & 2,246 & 0,445 & 4,156 & $-4,156$ & 3,845 & 0,260 & 1,504 & $-16599,635$ \\
\hline 4CPBI & $-6,467$ & $-2,028$ & 6,467 & 2,028 & 4,439 & 2,220 & 0,451 & 4,248 & $-4,248$ & 4,065 & 0,246 & 4,841 & $-29105,633$ \\
\hline 4MPBI & $-6,235$ & $-1,846$ & 6,235 & 1,846 & 4,390 & 2,195 & 0,456 & 4,041 & $-4,041$ & 3,719 & 0,269 & 0,928 & $-17668,758$ \\
\hline 4NPBI & $-8,178$ & $-5,203$ & 8,178 & 5,203 & 2,976 & 1,488 & 0,672 & 6,690 & $-6,690$ & 15,043 & 0,066 & 10,258 & $-3763,007$ \\
\hline 4APBI & $-7,004$ & $-3,391$ & 7,004 & 3,391 & 3,613 & 1,807 & 0,554 & 5,197 & $-5,197$ & 7,476 & 0,134 & 7,187 & $-2933,601$ \\
\hline 2NPBI & $-8,168$ & $-5,044$ & 8,168 & 5,044 & 3,124 & 1,562 & 0,640 & 6,606 & $-6,606$ & 13,968 & 0,072 & 5,275 & $-3762,659$ \\
\hline 2APBI & $-7,426$ & $-3,335$ & 7,426 & 3,335 & 4,091 & 2,045 & 0,489 & 5,381 & $-5,381$ & 7,077 & 0,141 & 6,980 & $-2934,065$ \\
\hline PBI & $-7,914$ & $-3,733$ & 7,914 & 3,733 & 4,182 & 2,091 & 0,478 & 5,824 & $-5,824$ & 8,111 & 0,123 & 2,767 & $-2649,691$ \\
\hline 4CPBI & $-7,968$ & $-3,940$ & 7,968 & 3,940 & 4,027 & 2,014 & 0,497 & 5,954 & $-5,954$ & 8,802 & 0,114 & 6,076 & $-3038,525$ \\
\hline 4APBI & $-5,384$ & $-1,216$ & 5,384 & 1,216 & 4,168 & 2,084 & 0,480 & 3,300 & $-3,300$ & 2,612 & 0,383 & 11,861 & $-18008,855$ \\
\hline 2NPBI & $-6,593$ & $-2,818$ & 6,593 & 2,818 & 3,775 & 1,887 & 0,530 & 4,706 & $-4,706$ & 5,866 & 0,170 & 5,702 & $-22043,682$ \\
\hline 2APBI & $-5,620$ & $-1,355$ & 5,620 & 1,355 & 4,265 & 2,133 & 0,469 & 3,487 & $-3,487$ & 2,851 & 0,351 & 6,313 & $-18009,045$ \\
\hline PBI & $-6,281$ & $-1,619$ & 6,281 & 1,619 & 4,662 & 2,331 & 0,429 & 3,950 & $-3,950$ & 3,346 & 0,299 & 1,734 & $-16510,766$ \\
\hline 4CPBI & $-6,391$ & $-1,785$ & 6,391 & 1,785 & 4,606 & 2,303 & 0,434 & 4,088 & $-4,088$ & 3,628 & 0,276 & 5,402 & $-28957,519$ \\
\hline 4MPBI & $-6,133$ & $-1,565$ & 6,133 & 1,565 & 4,567 & 2,284 & 0,438 & 3,849 & $-3,849$ & 3,244 & 0,308 & 0,297 & $-17574,226$ \\
\hline 4BPBI & $-6,261$ & $-1,731$ & 6,261 & 1,731 & 4,530 & 2,265 & 0,442 & 3,996 & $-3,996$ & 3,526 & 0,284 & 3,579 & $-86205,643$ \\
\hline \multicolumn{14}{|c|}{ B3LYP/6-31g } \\
\hline 4NPBI & $-6,601$ & $-3,273$ & 6,601 & 3,273 & 3,328 & 1,664 & 0,601 & 4,937 & $-4,937$ & 7,324 & 0,137 & 11,136 & $-22160,860$ \\
\hline 4APBI & $-5,421$ & $-1,262$ & 5,421 & 1,262 & 4,158 & 2,079 & 0,481 & 3,341 & $-3,341$ & 2,685 & 0,372 & 11,164 & $-18104,077$ \\
\hline 2NPBI & $-6,534$ & $-2,835$ & 6,534 & 2,835 & 3,699 & 1,849 & 0,541 & 4,684 & $-4,684$ & 5,932 & 0,169 & 5,584 & $-22160,389$ \\
\hline 2APBI & $-5,639$ & $-1,381$ & 5,639 & 1,381 & 4,258 & 2,129 & 0,470 & 3,510 & $-3,510$ & 2,893 & 0,346 & 5,802 & $-18104,128$ \\
\hline PBI & $-6,202$ & $-1,622$ & 6,202 & 1,622 & 4,581 & 2,290 & 0,437 & 3,912 & $-3,912$ & 3,341 & 0,299 & 1,437 & $-16598,127$ \\
\hline PBI & $-6,446$ & $-1,927$ & 6,446 & 1,927 & 4,519 & 2,259 & 0,443 & 4,186 & $-4,186$ & 3,878 & 0,258 & 1,385 & $-16598,804$ \\
\hline 4CPBI & $-6,522$ & $-2,049$ & 6,522 & 2,049 & 4,473 & 2,236 & 0,447 & 4,286 & $-4,286$ & 4,106 & 0,244 & 5,005 & $-29104,924$ \\
\hline 4MPBI & $-6,278$ & $-1,877$ & 6,278 & 1,877 & 4,401 & 2,200 & 0,454 & 4,078 & $-4,078$ & 3,778 & 0,265 & 1,185 & $-17667,850$ \\
\hline 4BPBI & $-6,477$ & $-2,044$ & 6,477 & 2,044 & 4,433 & 2,217 & 0,451 & 4,260 & $-4,260$ & 4,094 & 0,244 & 4,225 & $-86560,265$ \\
\hline \multicolumn{14}{|c|}{ B3LYP/Lanl2dz } \\
\hline 4NPBI & $-6,801$ & $-3,606$ & 6,801 & 3,606 & 3,195 & 1,598 & 0,626 & 5,203 & $-5,203$ & 8,473 & 0,118 & 11,813 & $-22163,791$ \\
\hline 4APBI & $-5,648$ & $-1,568$ & 5,648 & 1,568 & 4,080 & 2,040 & 0,490 & 3,608 & $-3,608$ & 3,191 & 0,313 & 11,672 & $-18105,916$ \\
\hline 2NPBI & $-6,738$ & $-3,263$ & 6,738 & 3,263 & 3,475 & 1,738 & 0,575 & 5,000 & $-5,000$ & 7,194 & 0,139 & 5,944 & $-22163,343$ \\
\hline 2APBI & $-5,858$ & $-1,668$ & 5,858 & 1,668 & 4,189 & 2,095 & 0,477 & 3,763 & $-3,763$ & 3,380 & 0,296 & 6,222 & $-18105,954$ \\
\hline PBI & $-6,408$ & $-1,913$ & 6,408 & 1,913 & 4,495 & 2,247 & 0,445 & 4,161 & $-4,161$ & 3,851 & 0,260 & 1,520 & $-16599,610$ \\
\hline 4CPBI & $-6,482$ & $-2,037$ & 6,482 & 2,037 & 4,444 & 2,222 & 0,450 & 4,259 & $-4,259$ & 4,082 & 0,245 & 5,016 & $-16990,077$ \\
\hline 4MPBI & $-6,240$ & $-1,849$ & 6,240 & 1,849 & 4,391 & 2,195 & 0,455 & 4,045 & $-4,045$ & 3,726 & 0,268 & 0,921 & $-17668,733$ \\
\hline 4BPBI & $-6,442$ & $-2,039$ & 6,442 & 2,039 & 4,403 & 2,202 & 0,454 & 4,241 & $-4,241$ & 4,084 & 0,245 & 4,386 & $-16941,636$ \\
\hline
\end{tabular}


Table 3. The calculated quantum chemical parameters with HF method in gas phase (eV)

\begin{tabular}{|c|c|c|c|c|c|c|c|c|c|c|c|c|c|}
\hline & Еномо & ELumo & I & $\mathbf{A}$ & $\Delta \mathbf{E}$ & $\eta$ & $\sigma$ & $\chi$ & $\mathbf{P} \dot{\mathbf{I}}$ & $\omega$ & $\varepsilon$ & dipol & Energy \\
\hline \multicolumn{14}{|l|}{ HF/SDD } \\
\hline 4APBI & $-7,518$ & 2,499 & 7,518 & $-2,499$ & 10,017 & 5,009 & 0,200 & 2,509 & $-2,509$ & 0,629 & 1,591 & 1,251 & $-17988,851$ \\
\hline 2APBI & $-7,286$ & 2,280 & 7,286 & $-2,280$ & 9,566 & 4,783 & 0,209 & 2,503 & $-2,503$ & 0,655 & 1,527 & 5,928 & $-17987,967$ \\
\hline PBI & $-8,307$ & 2,265 & 8,307 & $-2,265$ & 10,572 & 5,286 & 0,189 & 3,021 & $-3,021$ & 0,863 & 1,158 & 1,206 & $-16492,155$ \\
\hline 4CPBI & $-9,140$ & 0,209 & 9,140 & $-0,209$ & 9,349 & 4,675 & 0,214 & 4,466 & $-4,466$ & 2,133 & 0,469 & 4,423 & $-28979,512$ \\
\hline 4MPBI & $-8,148$ & 2,403 & 8,148 & $-2,403$ & 10,552 & 5,276 & 0,190 & 2,872 & $-2,872$ & 0,782 & 1,279 & 0,584 & $-17553,403$ \\
\hline \multicolumn{14}{|c|}{ HF/Cep-4g } \\
\hline 4NPBI & $-11,048$ & $-0,994$ & 11,048 & 0,994 & 10,054 & 5,027 & 0,199 & 6,021 & $-6,021$ & 3,606 & 0,277 & 7,817 & $-3662,891$ \\
\hline 4APBI & $-8,991$ & 1,491 & 8,991 & $-1,491$ & 10,481 & 5,241 & 0,191 & 3,750 & $-3,750$ & 1,342 & 0,745 & 0,917 & $-2849,671$ \\
\hline 2NPBI & $-11,022$ & 0,115 & 11,022 & $-0,115$ & 11,136 & 5,568 & 0,180 & 5,453 & $-5,453$ & 2,670 & 0,374 & 4,664 & $-3662,380$ \\
\hline 2APBI & $-8,604$ & 1,346 & 8,604 & $-1,346$ & 9,951 & 4,975 & 0,201 & 3,629 & $-3,629$ & 1,323 & 0,756 & 2,482 & $-2849,719$ \\
\hline PBI & $-10,075$ & 1,128 & 10,075 & $-1,128$ & 11,203 & 5,601 & 0,179 & 4,473 & $-4,473$ & 1,786 & 0,560 & 1,478 & $-2573,138$ \\
\hline 4CPBI & $-10,547$ & 0,580 & 10,547 & $-0,580$ & 11,127 & 5,564 & 0,180 & 4,984 & $-4,984$ & 2,232 & 0,448 & 4,665 & $-2955,887$ \\
\hline 4APBI & $-7,264$ & 2,858 & 7,264 & $-2,858$ & 10,122 & 5,061 & 0,198 & 2,203 & $-2,203$ & 0,479 & 2,086 & 0,472 & $-17893,170$ \\
\hline 2NPBI & $-9,416$ & 1,386 & 9,416 & $-1,386$ & 10,802 & 5,401 & 0,185 & 4,015 & $-4,015$ & 1,492 & 0,670 & 4,741 & $-21908,839$ \\
\hline 2APBI & $-7,310$ & 2,611 & 7,310 & $-2,611$ & 9,921 & 4,960 & 0,202 & 2,349 & $-2,349$ & 0,556 & 1,798 & 1,916 & $-17893,832$ \\
\hline PBI & $-8,354$ & 2,571 & 8,354 & $-2,571$ & 10,925 & 5,462 & 0,183 & 2,892 & $-2,892$ & 0,765 & 1,307 & 1,096 & $-16404,596$ \\
\hline 4CPBI & $-8,659$ & 2,177 & 8,659 & $-2,177$ & 10,836 & 5,418 & 0,185 & 3,241 & $-3,241$ & 0,969 & 1,032 & 4,153 & $-28832,859$ \\
\hline 4MPBI & $-8,181$ & 2,665 & 8,181 & $-2,665$ & 10,845 & 5,423 & 0,184 & 2,758 & $-2,758$ & 0,701 & 1,426 & 0,553 & $-17460,279$ \\
\hline 4BPBI & $-8,490$ & 2,259 & 8,490 & $-2,259$ & 10,749 & 5,375 & 0,186 & 3,115 & $-3,115$ & 0,903 & 1,108 & 3,201 & $-86052,485$ \\
\hline \multicolumn{14}{|c|}{ HF/6-31g } \\
\hline 4NPBI & $-9,507$ & 1,349 & 9,507 & $-1,349$ & 10,856 & 5,428 & 0,184 & 4,079 & $-4,079$ & 1,533 & 0,652 & 7,884 & $-22024,747$ \\
\hline 4APBI & $-7,504$ & 3,068 & 7,504 & $-3,068$ & 10,572 & 5,286 & 0,189 & 2,218 & $-2,218$ & 0,465 & 2,150 & 1,393 & $-17987,154$ \\
\hline 2NPBI & $-8,786$ & 1,942 & 8,786 & $-1,942$ & 10,727 & 5,364 & 0,186 & 3,422 & $-3,422$ & 1,092 & 0,916 & 4,966 & $-22023,629$ \\
\hline 2APBI & $-7,769$ & 2,864 & 7,769 & $-2,864$ & 10,633 & 5,316 & 0,188 & 2,453 & $-2,453$ & 0,566 & 1,768 & 1,602 & $-17987,206$ \\
\hline 2APBI & $-7,948$ & 1,053 & 7,948 & $-1,053$ & 9,001 & 4,501 & 0,222 & 3,447 & $-3,447$ & 1,320 & 0,757 & 1,618 & $-17987,709$ \\
\hline PBI & $-8,323$ & 1,056 & 8,323 & $-1,056$ & 9,379 & 4,689 & 0,213 & 3,633 & $-3,633$ & 1,408 & 0,710 & 1,159 & $-16491,100$ \\
\hline 4CPBI & $-8,557$ & 0,974 & 8,557 & $-0,974$ & 9,531 & 4,766 & 0,210 & 3,792 & $-3,792$ & 1,508 & 0,663 & 4,066 & $-28978,198$ \\
\hline 4MPBI & $-8,155$ & 1,084 & 8,155 & $-1,084$ & 9,239 & 4,619 & 0,216 & 3,535 & $-3,535$ & 1,353 & 0,739 & 0,536 & $-17552,232$ \\
\hline 4BPBI & $-8,506$ & 0,982 & 8,506 & $-0,982$ & 9,489 & 4,744 & 0,211 & 3,762 & $-3,762$ & 1,492 & 0,670 & 3,766 & $-86403,766$ \\
\hline \multicolumn{14}{|c|}{ HF/Lanl2dz } \\
\hline 4NPBI & $-9,686$ & 0,357 & 9,686 & $-0,357$ & 10,044 & 5,022 & 0,199 & 4,664 & $-4,664$ & 2,166 & 0,462 & 8,602 & $-22027,001$ \\
\hline 4APBI & $-7,512$ & 2,501 & 7,512 & $-2,501$ & 10,013 & 5,006 & 0,200 & 2,506 & $-2,506$ & 0,627 & 1,595 & 1,248 & $-17988,792$ \\
\hline 2NPBI & $-9,598$ & 0,989 & 9,598 & $-0,989$ & 10,587 & 5,293 & 0,189 & 4,305 & $-4,305$ & 1,750 & 0,571 & 5,061 & $-22026,572$ \\
\hline 2APBI & $-7,922$ & 2,486 & 7,922 & $-2,486$ & 10,407 & 5,204 & 0,192 & 2,718 & $-2,718$ & 0,710 & 1,409 & 1,681 & $-17988,964$ \\
\hline PBI & $-8,306$ & 2,262 & 8,306 & $-2,262$ & 10,568 & 5,284 & 0,189 & 3,022 & $-3,022$ & 0,864 & 1,157 & 1,210 & $-16492,099$ \\
\hline 4CPBI & $-9,153$ & 2,081 & 9,153 & $-2,081$ & 11,233 & 5,617 & 0,178 & 3,536 & $-3,536$ & 1,113 & 0,898 & 4,567 & $-16877,010$ \\
\hline 4MPBI & $-8,143$ & 2,404 & 8,143 & $-2,404$ & 10,546 & 5,273 & 0,190 & 2,869 & $-2,869$ & 0,781 & 1,281 & 0,587 & $-17553,340$ \\
\hline 4BPBI & $-9,042$ & 2,096 & 9,042 & $-2,096$ & 11,138 & 5,569 & 0,180 & 3,473 & $-3,473$ & 1,083 & 0,924 & 4,187 & $-16828,705$ \\
\hline
\end{tabular}


Table 4. The calculated quantum chemical parameters with HF method in aqueous phase (eV)

\begin{tabular}{|c|c|c|c|c|c|c|c|c|c|c|c|c|c|}
\hline & $\mathbf{E}_{\text {номо }}$ & ELUMO $_{\text {LU }}$ & $\mathbf{I}$ & $\mathbf{A}$ & $\Delta \mathbf{E}$ & $\eta$ & $\sigma$ & $\chi$ & PI & $\omega$ & $\varepsilon$ & dipol & Energy \\
\hline \multicolumn{14}{|l|}{ HF/SDD } \\
\hline 4NPBI & $-9,572$ & 0,857 & 9,572 & $-0,857$ & 10,428 & 5,214 & 0,192 & 4,358 & $-4,358$ & 1,821 & 0,549 & 9,390 & $-22028,075$ \\
\hline 4APBI & $-8,474$ & 2,114 & 8,474 & $-2,114$ & 10,587 & 5,294 & 0,189 & 3,180 & $-3,180$ & 0,955 & 1,047 & 2,646 & $-17989,032$ \\
\hline 2NPBI & $-9,607$ & 0,626 & 9,607 & $-0,626$ & 10,233 & 5,116 & 0,195 & 4,490 & $-4,490$ & 1,970 & 0,508 & 6,386 & $-22027,146$ \\
\hline 2APBI & $-8,186$ & 2,262 & 8,186 & $-2,262$ & 10,447 & 5,224 & 0,191 & 2,962 & $-2,962$ & 0,840 & 1,191 & 2,161 & $-17989,338$ \\
\hline PBI & $-8,525$ & 2,050 & 8,525 & $-2,050$ & 10,576 & 5,288 & 0,189 & 3,237 & $-3,237$ & 0,991 & 1,009 & 1,492 & $-16492,429$ \\
\hline 4CPBI & $-8,633$ & 1,922 & 8,633 & $-1,922$ & 10,555 & 5,277 & 0,189 & 3,355 & $-3,355$ & 1,067 & 0,937 & 4,965 & $-28979,199$ \\
\hline 4MPBI & $-8,392$ & 2,167 & 8,392 & $-2,167$ & 10,559 & 5,279 & 0,189 & 3,112 & $-3,112$ & 0,917 & 1,090 & 0,749 & $-17553,678$ \\
\hline 4BPBI & $-8,596$ & 1,864 & 8,596 & $-1,864$ & 10,460 & 5,230 & 0,191 & 3,366 & $-3,366$ & 1,083 & 0,923 & 4,650 & $-16834,047$ \\
\hline \multicolumn{14}{|c|}{ HF/Cep-4g } \\
\hline 4NPBI & $-11,038$ & $-1,211$ & 11,038 & 1,211 & 9,827 & 4,913 & 0,204 & 6,125 & $-6,125$ & 3,817 & 0,262 & 8,804 & $-3663,269$ \\
\hline 4APBI & $-8,517$ & 1,842 & 8,517 & $-1,842$ & 10,359 & 5,179 & 0,193 & 3,337 & $-3,337$ & 1,075 & 0,930 & 19,751 & $-2847,722$ \\
\hline 2NPBI & $-11,159$ & $-0,967$ & 11,159 & 0,967 & 10,192 & 5,096 & 0,196 & 6,063 & $-6,063$ & 3,607 & 0,277 & 5,968 & $-3662,986$ \\
\hline 2APBI & $-9,023$ & 0,973 & 9,023 & $-0,973$ & 9,996 & 4,998 & 0,200 & 4,025 & $-4,025$ & 1,621 & 0,617 & 2,910 & $-2850,076$ \\
\hline PBI & $-10,381$ & 0,826 & 10,381 & $-0,826$ & 11,207 & 5,604 & 0,178 & 4,777 & $-4,777$ & 2,036 & 0,491 & 1,638 & $-2573,449$ \\
\hline 4CPBI & $-10,770$ & 0,403 & 10,770 & $-0,403$ & 11,173 & 5,587 & 0,179 & 5,184 & $-5,184$ & 2,405 & 0,416 & 5,309 & $-2956,186$ \\
\hline 4MPBI & $-10,326$ & 0,937 & 10,326 & $-0,937$ & 11,262 & 5,631 & 0,178 & 4,695 & $-4,695$ & 1,957 & 0,511 & 0,767 & $-2752,114$ \\
\hline 4BPBI & $-10,176$ & 0,713 & 10,176 & $-0,713$ & 10,888 & 5,444 & 0,184 & 4,732 & $-4,732$ & 2,056 & 0,486 & 3,325 & $-2915,754$ \\
\hline \multicolumn{14}{|l|}{ HF/3-21g } \\
\hline 4NPBI & $-9,143$ & 508 & 9,143 & 0,508 & 9,651 & 4,826 & 0,207 & 4,318 & $-4,318$ & 1,931 & 0,518 & 8,571 & $-21909,680$ \\
\hline 4APBI & $-7,485$ & 2,568 & 7,485 & $-2,568$ & 10,053 & 5,027 & 0,199 & 2,458 & $-2,458$ & 0,601 & 1,663 & 1,165 & $-17894,129$ \\
\hline 2NPBI & $-9,442$ & 1,506 & 9,442 & $-1,506$ & 10,948 & 5,474 & 0,183 & 3,968 & $-3,968$ & 1,438 & 0,695 & 5,566 & $-21909,660$ \\
\hline 2APBI & $-8,609$ & 3,072 & 8,609 & $-3,072$ & 11,681 & 5,841 & 0,171 & 2,769 & $-2,769$ & 0,656 & 1,524 & 3,304 & $-17893,970$ \\
\hline PBI & $-8,607$ & 2,313 & 8,607 & $-2,313$ & 10,920 & 5,460 & 0,183 & 3,147 & $-3,147$ & 0,907 & 1,102 & 1,341 & $-16404,854$ \\
\hline 4CPBI & $-8,796$ & 2,030 & 8,796 & $-2,030$ & 10,826 & 5,413 & 0,185 & 3,383 & $-3,383$ & 1,057 & 0,946 & 5,007 & $-28833,117$ \\
\hline 4MPBI & $-9,120$ & 3,045 & 9,120 & $-3,045$ & 12,165 & 6,082 & 0,164 & 3,037 & $-3,037$ & 0,758 & 1,319 & 1,831 & $-17460,878$ \\
\hline 4BPBI & $-8,664$ & 2,083 & 8,664 & $-2,083$ & 10,747 & 5,373 & 0,186 & 3,291 & $-3,291$ & 1,008 & 0,992 & 3,817 & $-86052,729$ \\
\hline \multicolumn{14}{|l|}{ HF/6-31g } \\
\hline 4NPBI & 331 & 36 & 1 & 336 & 7 & 4,584 & 0,218 & 4,247 & $-4,247$ & 1,968 & 0,508 & 9,396 & $-22024,616$ \\
\hline 4APBI & $-7,350$ & 2,192 & 7,350 & $-2,192$ & 9,541 & 4,771 & 0,210 & 2,579 & $-2,579$ & 0,697 & 1,435 & 13,029 & $-17986,720$ \\
\hline 2NPBI & $-8,817$ & & & $-1,732$ & & 5,274 & & 3,542 & $-3,542$ & 1,189 & 0,841 & 6,228 & $-22024,133$ \\
\hline 2APBI & & & & $-2,633$ & & 5,3 & & 2,704 & $-2,704$ & 85 & & 2,063 & 498 \\
\hline PBI & & & & $-2,377$ & & 5,375 & & 2,998 & $-2,998$ & 36 & & 1,445 & 0,898 \\
\hline 4CPBI & & & & $-2,162$ & & 5,3 & & 3,180 & $-3,180$ & 77 & & 5,078 & 8,014 \\
\hline 4MPBI & $-8,241$ & & & $-2,455$ & & & & 2,893 & $-2,893$ & 32 & & 0,783 & 2,044 \\
\hline 4BPBI & $-8,469$ & 2,164 & 8,469 & $-2,164$ & 10,633 & 5,316 & 0,188 & 3,152 & $-3,152$ & 0,935 & 1,070 & 4,524 & $-86402,741$ \\
\hline \multicolumn{14}{|c|}{$\mathrm{HF} / 6-31++\mathrm{g}$} \\
\hline 4NPBI & $-8,942$ & $-0,031$ & 8,942 & 0,031 & 8,911 & 4,456 & 0,224 & 4,486 & $-4,486$ & 2,259 & 0,443 & 9,556 & $-22025,334$ \\
\hline 4APBI & $-7,956$ & 1,127 & 7,956 & $-1,127$ & 9,083 & 4,541 & 0,220 & 3,414 & $-3,414$ & 1,283 & 0,779 & 1,791 & $-17988,068$ \\
\hline 2NPBI & $-8,769$ & 1,250 & 8,769 & $-1,250$ & 10,018 & 5,009 & 0,200 & 3,760 & $-3,760$ & 1,411 & 0,709 & 0,050 & $-5258,296$ \\
\hline 2APBI & $-8,199$ & 1,136 & 8,199 & $-1,136$ & 9,335 & 4,667 & 0,214 & 3,532 & $-3,532$ & 1,336 & 0,748 & 2,086 & $-17988,013$ \\
\hline PBI & $-8,522$ & 1,118 & 8,522 & $-1,118$ & 9,640 & 4,820 & 0,207 & 3,702 & $-3,702$ & 1,422 & 0,703 & 1,465 & $-16491,364$ \\
\hline 4CPBI & $-8,639$ & 1,136 & 8,639 & $-1,136$ & 9,775 & 4,888 & 0,205 & 3,751 & $-3,751$ & 1,440 & 0,695 & 4,977 & $-28978,465$ \\
\hline 4MPBI & $-8,384$ & 1,137 & 8,384 & $-1,137$ & 9,521 & 4,760 & 0,210 & 3,623 & $-3,623$ & 1,379 & 0,725 & 0,731 & $-17552,497$ \\
\hline 4BPBI & $-6,477$ & $-2,044$ & 6477 & 2,044 & 4,433 & 2,217 & 0,451 & 4,260 & $-4,260$ & 4,094 & 0,244 & 4,225 & $-86560,265$ \\
\hline \multicolumn{14}{|c|}{ HF/Lanl2dz } \\
\hline 4NPBI & $-9,464$ & 0,170 & 9,464 & $-0,170$ & 9,633 & 4,817 & 0,208 & 4,647 & $-4,647$ & 2,242 & 0,446 & 10,080 & $-22027,451$ \\
\hline 4APBI & $-7,719$ & 2,243 & 7,719 & $-2,243$ & 9,962 & 4,981 & 0,201 & 2,738 & $-2,738$ & 0,753 & 1,329 & 0,565 & $-17989,775$ \\
\hline 2NPBI & $-9,598$ & 0,627 & 9,598 & $-0,627$ & 10,225 & 5,112 & 0,196 & 4,486 & $-4,486$ & 1,968 & 0,508 & 6,389 & $-22027,085$ \\
\hline 2APBI & $-7,820$ & 1,992 & 7,820 & $-1,992$ & 9,812 & 4,906 & 0,204 & 2,914 & $-2,914$ & 0,865 & 1,156 & 5,161 & $-17988,423$ \\
\hline PBI & $-8,518$ & 2,053 & 8,518 & $-2,053$ & 10,571 & 5,285 & 0,189 & 3,232 & $-3,232$ & 0,988 & 1,012 & 1,495 & $-16492,373$ \\
\hline 4CPBI & $-8,636$ & 1,915 & 8,636 & $-1,915$ & 10,551 & 5,276 & 0,190 & 3,360 & $-3,360$ & 1,070 & 0,934 & 5,144 & $-16876,701$ \\
\hline 4MPBI & $-8,384$ & 2,170 & 8,384 & $-2,170$ & 10,554 & 5,277 & 0,190 & 3,107 & $-3,107$ & 0,915 & 1,093 & 0,752 & $-17553,614$ \\
\hline 4BPBI & $-8,585$ & 1,891 & 8,585 & $-1,891$ & 10,475 & 5,238 & 0,191 & 3,347 & $-3,347$ & 1,069 & 0,935 & 4,679 & $-16828,383$ \\
\hline
\end{tabular}

Chemical hardness [20,22-24] is the resistance to electron cloud polarization or deformation of chemical species. Chemical hardness is a very important parameter that is investigating a reactivity of molecules in both experimental and theoretical chemistry.Global softness, $\Delta \mathrm{E}$, and chemical hardness are related to each other.In the light of information of Koopman's theory [23], both chemical hardness value and global softness value are taken place HOMO and LUMO energy value. If the hard molecules have high HOMOLUMO energy gap, this molecule is not a good corrosion inhibitor. This molecule can't very easy give electron of HOMO to metal. From the light of 
the result given in table 1,2,3, and 4, we can see the corrosion inhibitor ranking of chemical hardness value as: $4 \mathrm{NPBI}>4 \mathrm{APBI}>2 \mathrm{NPBI}>$ $2 \mathrm{APBI}>4 \mathrm{BPBI}>4 \mathrm{MPBI}>4 \mathrm{CPBI}>\mathrm{PBI}$ in $\mathrm{B} 31 \mathrm{yp}$ method with sdd and lanl2dz.

The electronegativity value of molecules is a parameter that helps to compare the reactivity of molecules. Value of this parameter is given to predict the electron transfer between the metal and inhibitor. The molecule that has high electronegativity value, this molecule is hardly given the valances electron. Because these electrons are attracted more than other molecules by the nucleus. According to Sanderson's electronegativity equalization [24-25], we are work out the value of electrons transferred from corrosion inhibitor molecule by the following equation:

$\Delta N_{\max }=\frac{\chi_{M}-\chi_{\operatorname{inh}}}{2\left(\eta_{M}+\eta_{\text {inh }}\right)}$

where $\chi_{M}$ and $\chi_{i n h}$ are electronegativity of metal and inhibitor molecule, respectively. $\eta_{M}$ and $\eta_{\text {inh }}$ are chemical hardness of inhibitor molecule and metal, respectively.

In all parameter, we can write that 4 NPBI is the best corrosion inhibitor more than another molecule. The activities of these molecules may be calculated using different programs [2627].Moreover, a similar ranking was given in the experimental study managed by Dutta and his coworker [1].

\section{CONCLUSION}

Benzimidazole derivatives were performed at Hartree-Fock and b3lyp with different basis set to investigate the corrosion inhibition activity.The result of quantum chemical parameters was shown that the corrosion inhibition efficiency ranking of these molecules can be givenas: 4NPBI $>4 \mathrm{APBI}>$ $2 \mathrm{NPBI}>2 \mathrm{APBI}>4 \mathrm{BPBI}>4 \mathrm{MPBI}>4 \mathrm{CPBI}>\mathrm{PBI}$ in B3lyp method with sdd and lanl2dz.From the light of the result given in table $1,2,3$, and 4 , these molecules are very important towards rational design new benzimidazole derivate as corrosion inhibitor.

\section{ACKNOWLEDGMENTS}

This research was made possible by TUBITAK ULAKBIM, High Performance and Grid Computing Center (TR-Grid e-Infrastructure).

\section{REFERENCES}

[1] Dutta, A., Saha, S. K., Adhikari, U., Banerjee, P., \& Sukul, D.,Effect of substitution on corrosion inhibition properties of 2-(substituted phenyl) benzimidazole derivatives on mild steel in $1 \mathrm{M} \mathrm{HCl}$ solution: a combined experimental and theoretical approach. Corrosion Science, 123 (2017) 256-266.

[2] Dennington R.D., Keith T.A., Millam J.M., Gauss View 5.0, 2009. Wallingford CT.

[3] Frisch M.J., Trucks G.W., Schlegel H.B., Scuseria G.E., Robb M.A., Cheseman J.R., Scalmani G., Barone V., Mennucci B., Petersson G.A., Nakatsuji H., Caricato H., Li X., Hratchian H.P., Izmaylov A.F., Bloino J., Zheng G., Sonnerberg J.L., Hada M., Ehara M., Toyota K., Fukuda R., Hasegawa J., Ishida M., Nakajima T., Honda Y., Kitao O., Nakai H., Vreven T., Montgomery J.A., Peralta J.E., Ogliaro F., Bearpark M., Heyd J.J., Brothers E., Kudin K.N., Staroverov V.N., Kobayashi R., Normand J., Raghavachari K., Rendell A., Burant J.C., Iyengar S.S., Tomasi J., Cossi M., Nega R., Millam J.M., Klene M., Knox J.E., Cross J.B., Bakken V., Adamo C., Jaramillo J., Gomperts R., Stratmann R.E., Yazyev O., Austin A.J., Cammi R., Pomelli C., Ochterski J.W., Martin R.L., Morokuma K., Zakrzewski V.G., Voth G.A., Salvador P., Dannenberg J.J., Daprich S., Daniels A.D., Farkas A., Foreaman J.B., Ortiz JV, Cioslowski J, Fox DJ. Gaussian 09, Revision D.01, Gaussian, Inc., WallingfordCT, 2009.

[4] Frisch M.J., Trucks G.W., Schlegel H.B., Scuseria G.E., Robb M.A., Cheseman J.R., Scalmani G., Barone V., Mennucci B., Petersson G.A., Nakatsuji H., Caricato H., Li X., Hratchian H.P., Izmaylov A.F., Bloino J., Zheng G., Sonnerberg J.L., Hada M., Ehara M., Toyota K., Fukuda R., Hasegawa J., Ishida M., Nakajima T., Honda Y., Kitao O., Nakai H., Vreven T., Montgomery J.A., Peralta J.E., Ogliaro F., Bearpark M., Heyd J.J., Brothers E., Kudin K.N., Staroverov V.N., Kobayashi R., Normand J., Raghavachari K., Rendell A., Burant J.C., Iyengar S.S., Tomasi J., Cossi M., Nega R., Millam J.M., Klene M., Knox J.E., Cross J.B., Bakken V., Adamo C., Jaramillo J., Gomperts R., Stratmann R.E., Yazyev O., Austin A.J., Cammi R., Pomelli C., Ochterski J.W., Martin R.L., Morokuma K., Zakrzewski V.G., Voth G.A., Salvador P., Dannenberg J.J., Daprich S., Daniels A.D., Farkas A., Foreaman J.B., Ortiz JV, Cioslowski J, Fox DJ., Gaussian 
09, Revision A.02, Gaussian, Inc., Wallingford CT, 2009.

[5] Becke A.D., A new mixing of Hatree-Fock and local density-functional theories, J.Chem. Phys., 98 (1993) 1372-1377.

[6] WibergK.B.. Basis set effects on calculated geometries: 6-311++G** vs. aug-cc-pVDZ. J. Comput. Chem., 25 (2004) 1342-1346.

[7] Beck A.D., Density-functional thermochemistry III. the role of exact, Exchange The Journal of Chemical Physics 98 (1993) 5648-5652.

[8] Lee C., Yang W., Parr R.G., Development of the Colle-Savletti correlation-energy formula into a functional of the electron density, Physical Review, 37 (1988) 785-789.

[9] Kaya S., Banerjee P., Saha S. K., Tüzün B., Kaya C., Theoretical evaluation of some benzotriazole and phospono derivatives as aluminum corrosion inhibitors: DFT and molecular dynamics simulation approaches, RSC Advances, 6 (2016) 74550-74559.

[10] Kaya S., Tüzün B., Kaya C., Obot I.B., Determination of corrosion inhibition effects of amino acids: Quantum chemical and molecular dynamic simulation study, Journal of the Taiwan Institute of Chemical Engineers, 58 (2016) 528-553.

[11] Kaya S., Kaya C., Guo L., Kandemirli F., Tüzün B., Uğurlu İ., Madkour L.H., Saraçoğlu M., Quantum chemical and molecular dynamics simulation studies on inhibition performances of some thiazole and thiadiazole derivatives against corrosion of iron. Journal of Molecular Liquids, 219 (2016) 497-504.

[12] Tüzün B., Selectivity of Salicylaldoxime and its Derivatives, Journal of New Results in Science, 3-5 (2014) 67-85.

[13] E. Kose, A. Atac, F. Bardak, the structural and spectroscopic investigation of 2-chloro-3methylquinoline by DFT method and UV-Vis, NMR and vibrational spectral techniques combined with molecular docking analysis. Journal of Molecular Structure, 1163 (2018) 147-160.

[14] Z. Zhang, W. Li, W. Zhang, X. Huang, L. Ruan, L. Wu, Experimental, quantum chemical calculations and molecular dynamics (MD) simulation studies of methionine and valine as corrosion inhibitors on carbon steel in phase change materials (PCMs) solution. Journal of Molecular Liquids, 272 (2018) 528-538.

[15] R. Nabah, F. Benhiba, Y. Ramli, M. Ouakki, M. Cherkaoui, H. Oudda, A. Zarrouk, Corrosion Inhibition Study of 5, 5-diphenylimidazolidine-
2, 4-dione for Mild Steel Corrosion in $1 \mathrm{M} \mathrm{HCl}$

Solution: Experimental, Theoretical Computational and Monte Carlo Simulations Studies. Analytical \& Bioanalytical Electrochemistry, 10-10 (2018) 1375-1398.

[16] M. Alam, D.U. Lee, Synthesis, spectroscopic and computational studies of 2-(thiophen-2-yl)2, 3-dihydro-1H-perimidine: An enzymes inhibition study. Computational Biology and Chemistry, 64 (2016) 185-201.

[17] Madkour L.H., Elshamy I.H. Experimental and computational studies on the inhibition performances of benzimidazole and its derivatives for the corrosion of copper in nitric acid. Int. J. Ind. Chem., 7 (2016) 195-221.

[18] Pearson RG. Chemical hardness: applications from molecules to solids. Germany: WileyVCH: Weinheim; 1997.

[19] Obot I.B., Obi-Egbedi N.O., Eselo A.O., Anticorrosion potential of 2-mesityl-1Himidazol[4,5-][1,10]-phenanthroline on mild steel in sulfuric acid solution; experimental and theoretical study, Ind. Eng. Chem. Res., 50 (2011) 2098-2110.

[20] Makov G., Chemical hardness in density functional theoryJ. Phys. Chem., 99 (1995) 9337-9339

[21] Pearson R.G., Hard and soft acids and bases, J. Am. Chem. Soc., 85 (1963) 3533-3539

[22] Pearson R.G., Hard and soft acids and bases, HSAB, part 1: Fundamental principles, J. Chem. Educ., 45 (1968) 581-587.

[23] Koopmans T., Ordering of wave functions and eigen-energies to the individual electrons of an atom, Physica, 1 (1993) 104-113.

[24] Sanderson R.T., Chemical bond and bond energy, Academic Press, New York 1976.

[25] Sanderson R.T., Electronegativities in inorganic chemistry, J. Chem. Educ., 31 (1954) 2-7.

[26] Özalp A.; Yavuz S. Ç.; Sabancı N.; Çopur F.; Kökbudak Z.; Sarıpınar E. 4D-QSAR investigation and pharmacophore identification of pyrrolo[2,1-c][1,4] benzodiazepines using electron conformational-genetic algorithm method. SAR and QSAR in Environmental Research, 27-4 (2016) 317-342.

[27] Tüzün B., Yavuz S. Ç., Sarıpınar E., 4D-QSAR analysis and pharmacophore modeling: propoxy methylphenyl oxasiazole derivatives by electron conformatitional-genetic algorithm method, Journal of Physical and Theoretical Chemistry of Islamic Azad University of Iran, 14-2 (2018) 149-164. 